\title{
PVC com condições de contorno Fuzzy: Solução baseada em Método de elementos finitos
}

\author{
Daniel Sánchez Ibáñez ${ }^{1}$ \\ Departamento de Matemática Aplicada, IMECC, UNICAMP, Campinas, SP \\ Laécio Carvalho de Barros ${ }^{2}$ \\ Departamento de Matemática Aplicada, IMECC, UNICAMP, Campinas, SP
}

Resumo. Nesse trabalho é estudado um problema de valor de contorno em que as condições de contorno são incertas e modeladas por números fuzzy. É apresentada uma solução numérica a partir do método de elementos finitos de Galerkin clássico. A solução fuzzy é obtida a partir de seus $\alpha$-níveis, que são intervalos da reta real. Um exemplo é apresentado para equação de Poisson unidimensional.

Palavras-chave. Problema de valor de contorno, método de elementos finitos, função fuzzy.

\section{Introdução}

Muitos problemas físicos em engenharia são modelados matematicamente a través de um problema de valor de contorno (PVC). De forma geral, um PVC é resolvido por métodos numéricos clássicos como o método de elementos finitos (MEF) de Galerkin [1,6]. É claro que tais modelos podem não ser fies ao fenômeno real representado, uma vez que considera valores numéricos exatos nas condições de contorno do problema mesmo que haja incerteza nos mesmos. Assim, nessa proposta, objetivou-se sugerir uma solução aproximada de um PVC quando tais condições de contorno são modeladas por números fuzzy [2]. Nesse trabalho, será considerado o seguinte PVC para a equação de Poisson [1,3] unidimensional, da forma

$$
\begin{cases}-\frac{d^{2} u}{d x^{2}}=f(x), & a<x<b, \\ u(a)=A, u(b)=B, & A \text { e } B \in \mathcal{F}(\mathbb{R}),\end{cases}
$$

onde as condições de contorno $A$ e $B$ são números fuzzy. De fato, propõe-se uma adaptação no espaço de funções "solução" requerida no MEF, de forma que elas sejam funções fuzzy [4] que representam a solução fuzzy do problema.

\footnotetext{
${ }^{1}$ daniel@ime.unicamp.br

2laeciocb@ime.unicamp.br
} 


\section{Conceitos preliminares}

Definição 2.1. [2] Seja U um conjunto (clássico); um subconjunto fuzzy $A$ de $U$ é caracterizado por uma função $\varphi_{A}: U \rightarrow[0,1]$, chamada função de pertinência.

Definição 2.2. [2] Seja $A$ um subconjunto fuzzy de $U$ e $\alpha \in[0,1]$. O $\alpha$-nível de $A$ é o subconjunto clássico de $U$ definido por $[A]^{\alpha}=\left\{x \in U: \varphi_{A}(x) \geq \alpha\right\}$ para $0<\alpha \leq 1$.

Para $\alpha=0$ temos $[A]^{0}=\overline{\operatorname{supp}} A$, isto é, o fecho do suporte de $A$, onde $\operatorname{supp} A=\{x \in$ $\left.\mathbb{R}: \varphi_{A}(x)>0\right\}$. Para $\alpha=1$ denotamos core $A=\left\{x \in \mathbb{R}: \varphi_{A}(x)=1\right\}[2,4]$.

Definição 2.3. [2] Um subconjunto fuzzy A é chamado de número fuzzy quando o conjunto universo no qual $\varphi_{A}$ está definida, é o conjunto dos números reais $\mathbb{R}$ e satisfaz às condições: (i) todos os $\alpha$-níveis de $A$ são não vazios, com $0 \leq \alpha \leq 1$; (ii) todos os $\alpha$-níveis de $A$ são intervalos fechados de $\mathbb{R}$; (iii) o suporte de $A$ é limitado.

Os $\alpha$-níveis do número fuzzy $A$ serão denotados numa forma paramétrica $[\underline{A}, \bar{A}]$, onde $\underline{A}$ e $\bar{A}$ são funções que representam o valor inferior e superior, respectivamente, do intervalo $[A]^{\alpha}$, para $\alpha \in[0,1][4,5]$.

Definição 2.4. [2,4] Um número fuzzy $A$ é dito triangular se sua representação paramétrica é da forma $[\underline{A}, \bar{A}]=[(m-a) \alpha+a,(m-b) \alpha+b]$, para todo $\alpha \in[0,1]$, onde $\operatorname{supp} A=[a, b]$ e $m=\operatorname{core} A$.

A notação de um número fuzzy triangular será denotada pela terna ordenada $(a ; m ; b)$ e a família dos números fuzzy será indicada por $\mathcal{F}(\mathbb{R})[2]$.

Definição 2.5. [4] Uma função com valores a número fuzzy, ou simplesmente função fuzzy, é o mapeamento $F:[a, b] \rightarrow \mathcal{F}(\mathbb{R})$, onde $[a, b] \subset \mathbb{R}$.

\section{Desenvolvimento}

Para resolver o PVC (1) é considerado que $u:[a, b] \rightarrow \mathcal{F}(\mathbb{R})$ é uma função fuzzy e que sua forma paramétrica é um par $[\underline{u}, \bar{u}]$ de funções, onde $\underline{u}(x, \alpha)$ e $\bar{u}(x, \alpha)$. Assim, a notação para o PVC é caracterizada em forma paramétrica [5] (extremos intervalares), para cada $\alpha \in[0,1]$, como :

$$
\left\{\begin{array}{l}
-\underline{u}^{\prime \prime}(x, \alpha)=f(x), a<x<b, \\
\underline{u}(a, \alpha)=\underline{A}, \underline{u}(b, \alpha)=\underline{B} . \\
-\bar{u}^{\prime \prime}(x, \alpha)=f(x), a<x<b \\
\bar{u}(a, \alpha)=\bar{A}, \bar{u}(b, \alpha)=\bar{B} .
\end{array}\right.
$$

O MEF de Galerkin permite resolver aproximadamente um PVC para a equação de Poisson unidimensional em forma clássica (isto é, com parâmetros e condições de contorno sendo números reais) e, nesse trabalho, será caracterizado em forma paramétrica (extremos intervalares). Primeiramente, multiplicamos a equação diferencial do problema por uma função "teste" $v=v(x)$ a qual pertence a um determinado espaço $V^{0}$ de funções [1]:

$$
V^{0}=\left\{v: \int_{a}^{b}\left(v(x)^{2}+v^{\prime}(x)^{2}\right) d x<\infty, v(a)=v(b)=0\right\} .
$$


Seguidamente, integramos em $x \in[a, b]$ e $\forall v \in V^{0}$, obtendo em forma paramétrica [1]:

$$
\begin{aligned}
& -\int_{a}^{b} \underline{u}^{\prime \prime}(x, \alpha) v(x) d x=\int_{a}^{b} f(x) v(x) d x, \\
& -\int_{a}^{b} \bar{u}^{\prime \prime}(x, \alpha) v(x) d x=\int_{a}^{b} f(x) v(x) d x .
\end{aligned}
$$

Logo, integrando por partes, e uma vez que $v \in V^{0}$, obtemos [1]:

$$
\begin{aligned}
& -\int_{a}^{b} \underline{u}^{\prime \prime}(x, \alpha) v(x) d x=\int_{a}^{b} \underline{u}^{\prime}(x, \alpha) v^{\prime}(x) d x-\underline{\left[u^{\prime}(x, \alpha) v(x)\right]_{a}^{b}}=\int_{a}^{b} \underline{u}^{\prime}(x, \alpha) v^{\prime}(x) d x, \\
& -\int_{a}^{b} \bar{u}^{\prime \prime}(x, \alpha) v(x) d x=\int_{a}^{b} \bar{u}^{\prime}(x, \alpha) v^{\prime}(x) d x-\left[\bar{u}^{\prime}(x, \alpha) v(x)\right]_{a}^{b}=\int_{a}^{b} \bar{u}^{\prime}(x, \alpha) v^{\prime}(x) d x .
\end{aligned}
$$

Agora, desde que $u(a)=A$ e $u(b)=B$ são condições de contorno sendo números fuzzy, precisamos soluções da forma $u:(\underline{u}(x, \alpha), \bar{u}(x, \alpha))$ para $0 \leq \alpha \leq 1$, no espaço:

$$
V=\left\{u:\left(\int_{a}^{b}\left(\underline{u}^{2}(x)+\underline{u}^{\prime 2}(x)\right) d x<\infty, \int_{a}^{b}\left(\bar{u}^{2}(x)+\bar{u}^{\prime 2}(x)\right) d x<\infty\right), u(a)=A, u(b)=B\right\} .
$$

Dessa forma, obtém-se a formulação variacional [1] paramétrica do problema (2), que é encontrar $u \in V$ que satisfaz, $\forall v \in V^{0}$ e para cada $\alpha \in[0,1]$ :

$$
\begin{aligned}
& \int_{a}^{b} \underline{u}^{\prime}(x, \alpha) v^{\prime}(x) d x=\int_{a}^{b} f(x) v(x) d x, \\
& \int_{a}^{b} \bar{u}^{\prime}(x, \alpha) v^{\prime}(x) d x=\int_{a}^{b} f(x) v(x) d x .
\end{aligned}
$$

Resolver o PVC (1), considerando a sua forma paramétrica (2), é equivalente [1] a resolver um problema em uma formulação variacional paramétrica (3), para cada $\alpha \in[0,1]$.

Desde que $u \in V$ (sendo $V$ um espaço de dimensão infinita), deseja-se construir uma solução aproximada $u_{h}$, em um espaço de dimensão finita $\left(V_{h}\right)$ [7]. Para tal fim, considerase a partição $\tau_{h}: a=x_{0}<x_{1}<\cdots<x_{n}<x_{n+1}=b$, do intervalo $[a, b]$ em subintervalos $I_{i}=\left(x_{i-1}, x_{i}\right)$, com comprimento $\left|I_{i}\right|=h_{i}=x_{i}-x_{i-1}$, para $i=1,2, \ldots, n+1$. Assim, os espaços discretos [1], para funções "solução" e "teste", podem-se definir como:

$$
\begin{gathered}
V_{h}=\left\{u_{h}:\left(\underline{u}_{h}(x, \alpha), \bar{u}_{h}(x, \alpha)\right), u_{h} \text { é função contínua sobre } \tau_{h}, u_{h}(a)=A, u_{h}(b)=B\right\}, \\
V_{h}^{0}=\left\{v=v_{h}(x), v_{h} \text { é função contínua sobre } \tau_{h}, v_{h}(a)=v_{h}(b)=0\right\} .
\end{gathered}
$$

Assim, a formulação de elementos finitos [7] paramétrica (a formulação variacional paramétrica (3) discreta) é encontrar $u_{h} \in V_{h}$ que satisfaz, $\forall v \in V_{h}^{0}$ e para cada $\alpha \in[0,1]$ :

$$
\begin{aligned}
& \int_{a}^{b} \underline{u}_{h}^{\prime}(x, \alpha) v^{\prime}(x) d x=\int_{a}^{b} f(x) v(x) d x, \\
& \int_{a}^{b} \bar{u}_{h}^{\prime}(x, \alpha) v^{\prime}(x) d x=\int_{a}^{b} f(x) v(x) d x .
\end{aligned}
$$

Agora, pode-se inferir que $u_{h}:\left(\underline{u}_{h}(x, \alpha), \bar{u}_{h}(x, \alpha)\right)$ é combinação linear de funções de base $\phi_{j}=\xi_{j} \varphi_{j}(x)$, do espaço $V_{h}$ onde $\xi_{j} \in \mathcal{F}(\mathbb{R})$, para $j=0,1, \ldots, n+1$, ou seja:

$$
\left(\underline{u}_{h}(x, \alpha), \bar{u}_{h}(x, \alpha)\right)=\left(\sum_{j=0}^{n+1} \underline{\xi}_{j} \varphi_{j}(x), \sum_{j=0}^{n+1} \bar{\xi}_{j} \varphi_{j}(x)\right) .
$$


Logo, e dado que $v \in V_{h}^{0}$, escolhemos $v(x)=\varphi_{i}(x)$ para $i=1, \ldots, n$. Assim, colocando com (5) e ordenando em (4), temos $\forall v \in V_{h}^{0}$ e para cada $\alpha \in[0,1]$ :

$$
\begin{aligned}
& \sum_{j=0}^{n+1} \underline{\xi}_{j}\left(\int_{a}^{b} \varphi_{j}^{\prime}(x) \varphi_{i}^{\prime}(x) d x\right)=\int_{a}^{b} f(x) \varphi_{i}(x) d x, \\
& \sum_{j=0}^{n+1} \bar{\xi}_{j}\left(\int_{a}^{b} \varphi_{j}^{\prime}(x) \varphi_{i}^{\prime}(x) d x\right)=\int_{a}^{b} f(x) \varphi_{i}(x) d x .
\end{aligned}
$$

Isto resulta em um problema discreto $[1,7]$ representado por um sistema de equações paramétrico, que em forma matricial fica expressado pelos sistemas lineares

$$
M \underline{\xi}=\underline{d} \quad \text { e } \quad M \bar{\xi}=\bar{d},
$$

em que, a matriz (de rigidez) $M=\left\{m_{i j}\right\}_{i, j=1}^{n}$ com $M \in \mathbb{R}^{n \times n}$, onde

$$
m_{i j}=\int_{a}^{b} \varphi_{j}^{\prime}(x) \varphi_{i}^{\prime}(x) d x,
$$

e os vetores $\underline{\xi}=\left\{\underline{\xi}_{j}\right\}_{j=1}^{n}, \bar{\xi}=\left\{\bar{\xi}_{j}\right\}_{j=1}^{n}, \underline{d}=\left\{\underline{d}_{i}\right\}_{i=1}^{n}$ e $\bar{d}=\left\{\bar{d}_{i}\right\}_{i=1}^{n}$, com $\underline{\xi}, \bar{\xi}, \underline{d}$ e $\bar{d} \in \mathbb{R}^{n \times 1}$, onde

$$
\begin{aligned}
& \underline{d}_{i}=\int_{a}^{b} f(x) \varphi_{i}(x) d x-\left(\underline{\xi}_{0} \int_{a}^{b} \varphi_{i}^{\prime}(x) \varphi_{0}^{\prime}(x) d x+\underline{\xi}_{n+1} \int_{a}^{b} \varphi_{i}^{\prime}(x) \varphi_{n+1}^{\prime}(x) d x\right), \\
& \bar{d}_{i}=\int_{a}^{b} f(x) \varphi_{i}(x) d x-\left(\bar{\xi}_{0} \int_{a}^{b} \varphi_{i}^{\prime}(x) \varphi_{0}^{\prime}(x) d x+\bar{\xi}_{n+1} \int_{a}^{b} \varphi_{i}^{\prime}(x) \varphi_{n+1}^{\prime}(x) d x\right) .
\end{aligned}
$$

Cabe ressaltar que a resolução de ambos os sistemas matriciais (6), para cada $\alpha \in[0,1]$, forneceram, a cada vez, um vetor de coeficientes $[\xi, \bar{\xi}]$ que estabelecem a representação paramétrica (por $\alpha$-níveis) da solução fuzzy (aproximada) $u_{h}$ em (5).

\section{Exemplo e Resultados}

Seja considerado o PVC (1) com $a=0, b=1, f(x)=x, A=(-0.1 ; 0 ; 0.1)$ e $B=$ $(-0.01 ; 0 ; 0.01)$, ou seja, com condições de contorno números fuzzy triangulares:

$$
\left\{\begin{array}{l}
-\frac{d^{2} u}{d x^{2}}=x, \quad 0<x<1 \\
u(0)=(-0.1 ; 0 ; 0.1) \\
u(1)=(-0.01 ; 0 ; 0.01)
\end{array}\right.
$$

Logo, considerando uma partição homogênea, isto é, para $h=h_{i}$, introduz-se as seguintes funções (chapéus) de base $\varphi_{j}$, para $j=1, \ldots, n[1,7]$ :

$$
\varphi_{j}(x)=\left\{\begin{array}{ll}
\frac{x-x_{j-1}}{h}, & x_{j-1} \leq x \leq x_{j} \\
\frac{x_{j+1}-x}{h}, & x_{j} \leq x \leq x_{j+1} \\
0, & \text { caso contrário }
\end{array},\right.
$$

e para os contornos $(j=0$ e $j=n+1)$ :

$$
\varphi_{0}(x)=\left\{\begin{array}{ll}
\frac{x_{1}-x}{h}, & x_{0} \leq x \leq x_{1} \\
0, & \text { caso contrário }
\end{array} \quad \text { e } \quad \varphi_{n+1}(x)=\left\{\begin{array}{ll}
\frac{x-x_{n}}{h}, & x_{n} \leq x \leq x_{n+1} \\
0, & \text { caso contrário }
\end{array} .\right.\right.
$$


Para a resolução dos sistemas (6) devemos achar os valores de todas as integrais anteriormente apresentadas em (7) e (8). Então, nos elementos da matriz $M(7)$, derivando as funções de base utilizadas (10) e integrando, para qualquer $\alpha \in[0,1]$, obtemos:

$$
\left\{m_{i j}\right\}= \begin{cases}m_{i i}=\frac{2}{h}, & \forall i=1,2, \ldots, n \\ m_{i, j-1}=m_{i-1, j}=\frac{-1}{h}, & \forall i, j=2, \ldots, n . \\ m_{i j}=0, & \text { para }|i-j|>1 .\end{cases}
$$

Supondo que $f\left(x_{i}\right)=f_{i}$ seja uma aproximação constante por partes de $f(x)$ em cada elemento $I_{i}$ da partição $\tau_{h}$, podemos obter os vetores $\underline{d}$ e $\bar{d}$ utilizando a aproximação de Taylor para $f_{i+1}=f_{i}+\mathcal{O}(h)$, em funções suficientemente suaves. Assim, os elementos dos vetores (8) (de carga), por cada $\alpha \in[0,1]$, são:

$$
\left\{\underline{d}_{i}, \bar{d}_{i}\right\}=\left\{\begin{array}{lll}
\underline{d}_{1}=h f_{1}+\frac{\xi_{0}}{h}, & \bar{d}_{1}=h f_{1}+\frac{\bar{\xi}_{0}}{h}, & i=1 . \\
\underline{d}_{i}=h f_{i}, & \bar{d}_{i}=h f_{i}, & \forall i=2, \ldots, n-1 . \\
\underline{d}_{n}=h f_{n}+\frac{\xi_{n+1}}{h}, & \bar{d}_{n}=h f_{n}+\frac{\bar{\xi}_{n+1}}{h}, & i=n .
\end{array}\right.
$$

Cabe destacar que, nesse exemplo e pelas funções de base utilizadas (10) e (11), cada um dos dois sistemas (6) terá solução única, para cada $\alpha \in[0,1]$, uma vez que $M$ se trata de uma matriz (12) tridiagonal, simétrica e definida positiva, o que a torna não singular.

Também, observa-se que os efeitos das condições de contorno fuzzy ficam evidenciados na primeira e última linha dos vetores de carga (13).

Os $\alpha$-níveis de $A=(-0.1 ; 0 ; 0.1)$ e de $B=(-0.01 ; 0 ; 0.01)$ são $[\underline{A}, \bar{A}]=[0.1(\alpha-$ $1), 0.1(1-\alpha)]$ e $[\underline{B}, \bar{B}]=[0.01(\alpha-1), 0.01(1-\alpha)]$, respectivamente. Assim, de $(2),(5)$ e pelas funções de base utilizadas (11), ficam determinados os $\alpha$-níveis de $\xi_{0}$ e $\xi_{n+1}$ :

$$
\begin{aligned}
& \underline{u}_{h}(0, \alpha)=\underline{\xi}_{0} \varphi_{0}(0)=\underline{\xi}_{0}=\underline{A}=0.1(\alpha-1), \\
& \bar{u}_{h}(0, \alpha)=\bar{\xi}_{0} \varphi_{0}(0)=\bar{\xi}_{0}=\bar{A}=0.1(1-\alpha), \\
& \underline{u}_{h}(1, \alpha)=\underline{\xi}_{n+1} \varphi_{n+1}(1)=\underline{\xi}_{n+1}=\underline{B}=0.01(\alpha-1), \\
& \bar{u}_{h}(1, \alpha)=\bar{\xi}_{n+1} \varphi_{n+1}(1)=\bar{\xi}_{n+1}=\bar{B}=0.01(1-\alpha) .
\end{aligned}
$$

A titulo de ilustração, vamos supor que $f(x)=x$ e considerar uma aproximação constante por partes de $f(x)$ em cada elemento da partição da forma $f\left(x_{i}\right)=f_{i}=i h$, para $i=1, \ldots, n$. A figura 1 representa a solução fuzzy de (9) a partir de (5), onde a parte mais escura representa maior pertinência (maior $\alpha$ ).

A figura 2 apresenta, tridimensionalmente, a solução fuzzy de (9) a partir de (5), onde a parte mais escura representa maior pertinência (maior $\alpha$ ), e a solução determinística (analítica exata) supondo contornos reais $A=B=0$. Como pode ser notado, a solução fuzzy que tem grau de pertinência máximo $(\alpha=1)$ é a trajetória que melhor se aproxima da solução determinística e, por esta razão, é chamada de preferida. 


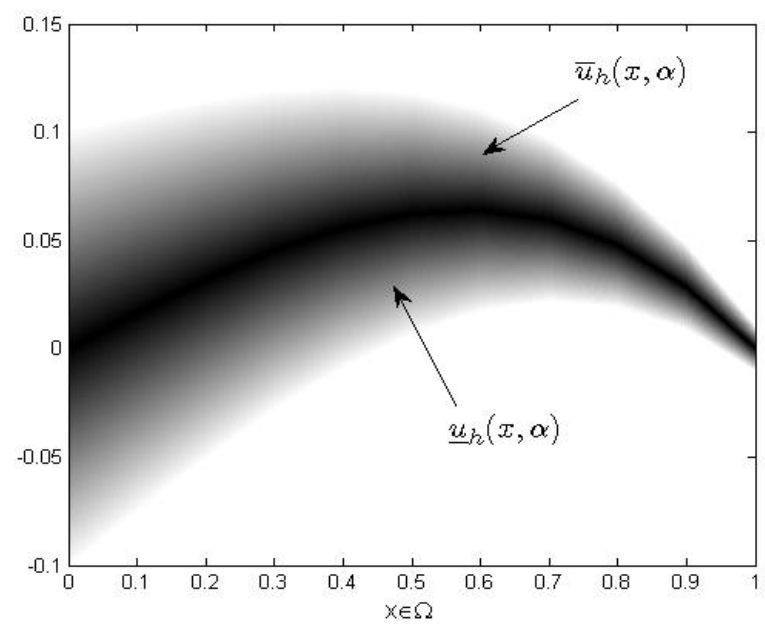

Figura 1: Solução fuzzy de (9) para $f(x)=x$, via (5) (baseado em MEF de Galerkin), com $A=(-0.1 ; 0 ; 0.1)$ e $B=(-0.01 ; 0 ; 0.01)$, onde a região mais escura indica maior $\alpha$.

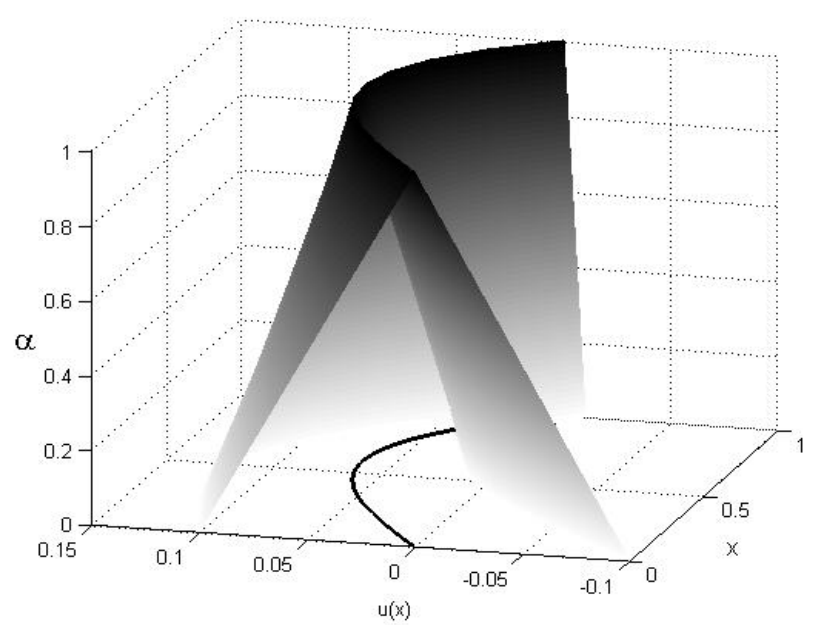

Figura 2: Solução determinística (preto) de (9) para $f(x)=x$, considerando $A=0$ e $B=0$, e solução (tridimensional) fuzzy, via (5) (baseado em MEF de Galerkin), com $A=(-0.1 ; 0 ; 0.1)$ e $B=(-0.01 ; 0 ; 0.01)$, onde região mais escura indica maior $\alpha$.

\section{Conclusões}

Neste trabalho vimos que é possível adotar o Método de elementos finitos (MEF) de Galerkin para obter uma solução fuzzy para um problema de valor de contorno (PVC) com condições de contorno dados por números fuzzy.

O tratamento matemático realizado é basicamente o tradicional (isto é, derivadas, integração e resolução de sistemas lineares de forma clássica) enquanto os números fuzzy são dados por seus $\alpha$-níveis para cada $\alpha \in[0,1]$. Este ordenamento estabelece estender o 
PVC original, numa representação paramétrica, inferior e outra superior, de cada intervalo e para cada $\alpha \in[0,1]$. Tal extensão é levada adiante e tratada tal como a teoria do MEF de Galerkin tradicional estabelece.

Uma vez que as condições de contorno são números fuzzy, inferiu-se que a solução do PVC deve ser uma função a valores de números fuzzy, isto é, $u:[a, b] \rightarrow \mathcal{F}(\mathbb{R})$ com $u:(\underline{u}(x, \alpha), \bar{u}(x, \alpha))$. Assim, as funções de base do espaço "solução" foram estabelecidas como funções reais multiplicadas por coeficientes sendo números fuzzy, tornando-se isto a maior modificação do MEF de Galerkin tradicional (isto é, uma extensão que considera funções fuzzy).

Finalmente, os gráficos da solução fuzzy ilustram dupla interpretações. Em primeiro lugar, como um conjunto de trajetórias determinísticas, com domínio $x \in[0,1]$ do problema, que limitam inferior e superiormente à solução determinística (quando os contornos são números reais), cuja melhor aproximação (a solução preferida) acontece no grau de pertinência máximo $(\alpha=1)$. Em segundo lugar, a solução fuzzy pode ser vista como função fuzzy, a qual fornece um número fuzzy para cada $x$ (fixo) do domínio, $\forall \alpha \in[0,1]$.

\section{Agradecimentos}

O segundo autor, L. C. de Barros, agradece ao CNPq processo 305862/2013-8.

\section{Referências}

[1] M. Asadzadeh. An Introduction to the Finite Element Method (FEM) for Differential Equations, 2014. DOI: 10.13140/2.1.4865.3128.

[2] L. C. Barros, R. C Bassanezi, and W. A. Lodwick. A First Course in Fuzzy Logic, Fuzzy Dynamical Systems, and Biomathematics. Theory and Applications. In Studies in Fuzziness and Soft Computing. Springer, 2017.

[3] A. M. Bertone, R. M. Jafelice, L. C. Barros, and R. C Bassanezi. On fuzzy solutions for partial differential equations, Fuzzy Sets and Systems, 219:68-80, 2013.

[4] L. T. Gomes, L. C. de Barros, and B. Bede. Fuzzy differential equation in various approaches. In SpringerBriefs in Mathematics. SBMAC - Springer, 2015. ISSN: 21918198.

[5] X. Guo, D. Shang, and X. Lu. Fuzzy approximate solutions of second-order fuzzy linear boundary value problems, Boundary Value Problems Journal, 2013:212, 2013.

[6] D. Ibanez, L. Bassani, L. Barros, and E. Laureano. Ensaios do Método de Elementos Finitos com Integral Fuzzy, In Recentes Avanços em Sistemas Fuzzy (Proceedings do IV CBSF). SBMAC, 2016. ISBN: 978-85-8215-079-5.

[7] J. Li, and Y. Chen. Computational Partial Differential Equations Using MATLAB. In Chapman \& Hall/CRC Applied Mathematics and Nonlinear Science Series, CRC Press, 2008. 